\title{
ESTRATEGIAS METODOLÓGICAS PARA LA ENSEÑANZA DE LA ORACIÓN DE RELATIVO A ESTUDIANTES DE ESPAÑOL COMO SEGUNDA LENGUA
}

\author{
Flor Garita Hernández.
}

\begin{abstract}
RESUMEN
En este trabajo se desarrollan algunas estrategias metodológicas para la enseñanza de la oración de relativo en español como segunda lengua. Su objetivo principal es brindarle al profesor de español la aplicación de una estrategia metodológica a la luz de las teorías y métodos modernos de enseñanza de segundas lenguas. Se ha escogido la oración de relativo porque es una de las estructuras catalogadas como un universal lingüístico que, como tal y de acuerdo con las teorías de Chomsky, estará presente en la mente de todos los estudiantes aunque hablen lenguas diferentes.
\end{abstract}

\begin{abstract}
This article develops certain methodological strategies for the teaching of the relative clause in Spanish as a Second Language. Its primary objective is to offer to the instructor of Spanish, the application of a methodological strategy in light of the modern pedagogical theories and methods of Second Language Acquisition and Teaching. The expression of relative clauses has been chosen because it is one of the structures classified as universal, and, in accordance with Chomsky's theories, will be present in the minds of all learners no matter their language background.
\end{abstract}

\section{Introducción}

En este trabajo se desarrollarán algunas estrategias metodológicas para la enseñanza de la oración de relativo en español como segunda lengua. Su objetivo principal es brindarle al profesor de español la aplicación de una estrategia metodológica a la luz de las teorías y métodos modernos de enseñanza de segundas lenguas. Se ha escogido la oración de relativo porque es una de las estructuras catalogadas como un universal lingüístico que, como tal y de acuerdo con las teorías de Chomsky, estará presente en la mente de todos los estudiantes aunque hablen lenguas diferentes.

De acuerdo con la teorías de los universales lingüísticos, la oración de relativo constituye uno de estos universales, ya que se encuentra presente en todas las lenguas, aunque con diferentes estructuras, según el tipo de lengua del que se trate. Para este estudio se seguirán los postulados de Keenan y Comrie en lo referente a los tipos de oración de relativo, citados por Cook (1996), además de lo que plantean los gramáticos de la lengua 
española, entre los que se destacan la Academia de la Lengua y Bosque y Demonte (2000). Para la parte metodológica se seguirán las propuestas de Bonnie Adair-Hauck (1994) y otros, y Scott Thornbury (2002)

\section{2. ¿Por qué enseñar gramática?}

De acuerdo con Harmer (1997:1), gramática "is the way in which words change themselves and group together to make sentences. The grammar of a language is what happens to words when they become plural or negative or what word order is used when we make questions or join two clauses to make one sentence”. Sin embargo, no sólo son las formas ni la manera como se unen las palabras en oraciones. La enseñanza de la gramática implica tomar en cuenta el significado y la función; es decir, el significado y el uso o los usos de esa estructura que estamos estudiando. El profesor debe conocer muy bien los tres aspectos: forma, significado y función y decidir cuántas o cuáles estructuras gramaticales va a estudiar y, de esas estructuras, cuáles significados va a enfatizar. Como señalan muy bien Celce-Murcia y Hilles (Celce-Murcia:123), "para que los profesores de segundas lenguas puedan presentar consistentemente la gramática y que sirva a un objetivo más alto nunca debe ser enseñada como un fin en sí misma, sino siempre con referencia al significado, a los factores sociales, al discurso o a la combinación de todos estos factores". En nuestro caso, de acuerdo con la teoría de la jerarquización (Comrie 1981), aparecen tres estructuras. Eso es suficiente para una clase. En la siguiente clase, se retoman las demás.

Antes del advenimiento del enfoque comunicativo, la gramática ocupó un lugar privilegiado en la enseñanza de segundas lenguas; con las teorías de la adquisición, principalmente con Krashen, se empezó a cuestionar ese papel privilegiado de la gramática hasta llegar a restarle importancia. Krashen, citado por Thornbury (2002), establece la diferencia entre aprender y adquirir una lengua. De acuerdo con este autor, aprender es el resultado de la enseñanza formal, generalmente de la gramática y es de un uso limitado para la comunicación real. La adquisición es un proceso natural: es el proceso por medio del cual se adquiere la primera lengua y por el que muchas otras lenguas se adquieren solo con el simple contacto con los hablantes de esas lenguas. La adquisición, según Krashen, ocurre cuando el aprendiz se expone al input correcto en un ambiente sin estrés. El éxito en una segunda lengua se debe a la adquisición, no al aprendizaje.

Con el enfoque comunicativo, no es que no se enseñara gramática sino que el eje vertebral de la enseñanza de segundas lenguas no lo constituía la gramática. El problema se genera, quizá, por la delimitación entre adquisición y aprendizaje de una segunda lengua. Según Krashen, para adquirir una segunda lengua no hace falta la explicación explícita de la gramática. Él tiene razón si se compara la adquisición de una segunda lengua con la adquisición de la primera, pero hasta qué punto el individuo domina esa primera lengua. Realmente lo que se hace es aprender a hablar lengua materna; pero si no hay instrucción gramatical, el individuo que habla esa lengua tendrá un vocabulario limitado, una pronunciación y una gramática deficientes. Si solamente se tratara de aprender a hablar sería igual que aprender a hablar la lengua materna; no obstante, un individuo, una vez que sabe hablar, empieza a recibir instrucción en el uso de su lengua materna formal e informalmente casi durante toda su vida, para lograr un dominio de las estructuras, ampliar el vocabulario y pronunciar adecuadamente, ser coherente en su discurso y muchas otras habilidades más, de acuerdo con los patrones de la sociedad en que vive. Lo mismo sucede con la adquisición de una segunda lengua: una 
persona puede aprender a hablar y a automatizar la lengua pero no tiene ni la conciencia de su funcionamiento ni las reglas para poder corregir sus propios errores.

Aprender una segunda lengua implica una formación en el uso correcto de las reglas tanto de las gramaticales como de pronunciación y un dominio del vocabulario. Vivian Cook (1996:14) al respecto afirma:

\footnotetext{
El conocimiento de la gramática es considerado por muchos lingüistas como el área central del sistema de lenguas alrededor del cual giran otras áreas como la pronunciación y el vocabulario (...) En alguna forma, la gramática es más fácil de estudiar para aprendices de segundas lenguas que otros aspectos de la lengua porque es altamente sistemática y sus efectos son, a menudo, obvios para sus hablantes.
}

Según Thornbury, en los últimos años se ha experimentado un reavivamiento de la gramática al surgir dos conceptos teóricos muy influyentes: el enfoque en la forma y el levantamiento o elevación de conciencia. La investigación sugiere que sin ninguna atención a la forma, los estudiantes corren el riesgo de la fosilización. Señalar las características del sistema gramatical es entonces una forma de hacer conciencia o de elevar la conciencia, aunque puede que no lleve directa e instantáneamente a la adquisición de estos elementos; sin embargo, puede desencadenar una serie de procesos mentales que en un momento van a dar como resultado la precisión y la producción apropiadas. Relacionado con la noción del levantamiento de la conciencia Krashen, citado por Thornbury, argumenta que la adquisición es un proceso inconsciente.

Hay posiciones a favor y en contra de la enseñanza de la gramática. Por ahora, interesa fundamentalmente destacar algunos de los argumentos en pro de la enseñanza de la gramática que plantea Thornbury (1999: 15).

$1 \quad$ "The sentence-machine argument"

Es el argumento de lo que algunos han llamado "item-learning", es decir, aprender un elemento. La gramática permite aprender oraciones y frases que se pueden memorizar al estilo de una máquina de hacer oraciones. La cantidad de oraciones posibles está determinada por el vocabulario que tiene el aprendiz y su creatividad.

1 "The fine-tuning argument"

La gramática comunica significados de tipo preciso. Cuando no hay un contexto o este no está claro, la gramática afina precisamente lo que no está claro en el contexto, mediante la conjugación de los verbos, con el sujeto de la oración, la concordancia, con los complementos verbales, etc. De esta forma, la gramática sirve no solo para autocorregirse sino también para aclarar ambigüedades, es decir, afina el significado, lo puntualiza.

1 "The fossilisation argument"

Si no se estudia la gramática, los errores se fosilizan, pues el aprendiz no tiene un modelo de corrección. Al respecto, Thornbury aclara:'Research suggest that learners who receive no instruction seem to be at risk of fossilising sooner than those who receive instruction" (1999:16). 
$1 \quad$ "The advance-organiser argument"

La gramática constituye un "organizador avanzado". Con esto, el autor nos explica que muchas veces hemos aprendido estructuras que no vamos a utilizar en un contexto inmediato; sin embargo, esas estructuras están ahí en la mente y en determinado momento, cuando logramos oírlas, las identificamos con claridad pues están almacenadas, es decir, no son extrañas para los aprendices.

$1 \quad$ "The discrete-item argument"

A diferencia de la enseñanza por situaciones o funciones comunicativas, la enseñanza por medio de la gramática permite separar ciertos elementos para estudiarlos mejor, es decir, diferenciarlos, asimilarlos y evaluarlos individualmente; tal es el caso de los pronombres, artículos y otras clases de palabras.

$1 \quad$ "The rule-of-low argument"

La gramática es un sistema de reglas que se le ofrecen al profesor para que las trasmita a los aprendices. La gramática es un sistema estructurado que puede ser aprendido y evaluado en pasos metodológicos. Para Westney, una de las tareas de la gramática pedagógica es la formulación de reglas (1994: 72). Esas reglas deben ser concretas, simples, no técnicas, acumulativas, cercanas a las tradiciones populares o tradicionales y en forma de regla general. Es importante tener en cuenta, también, que no hay una regla que sea la mejor. Tanto el profesor como los estudiantes deben asumir una actitud cautelosa hacia cualquier tratamiento pedagógico de la regularidad de una lengua.

1 "The learner expectations argument"

Muchos estudiantes llegan a las clases de segunda lengua con la expectativa de que la enseñanza será enfocada en la gramática. Estos estudiantes pueden haberse matriculado específicamente para asegurar que la experiencia de aprendizaje se haga más eficiente y sistemática.

Por todas estas razones, la gramática no puede ser ignorada en una clase de segunda lengua.

\section{La oración de relativo en español}

Se entiende por oración de relativo, en español, aquella oración subordinada que va introducida por un pronombre relativo y que funciona como un adjetivo. En este estudio, solo vamos a trabajar con los verdaderos pronombres relativos, no con los adverbios que funcionan como tales en algunas oraciones subordinadas. Los pronombre relativos constituyen el nexo de la oración subordinada o dependiente y se refieren a un antecedente que puede estar presente o no en la oración. Los pronombres relativos son los siguientes:

Que: pronombre invariable en cuanto a su morfología y se usa en función anafórica tanto para referirse a personas como a cosas. 
Quien: es un pronombre que varía en cuanto al número, pero tiene la restricción de que solo se usa con personas en dos funciones principales: introduce una subordinada de generalización equivalente a el y la que, los y las que. Su otra función está determinada por una preposición con la que expresa diferentes significados. Casi todas las preposiciones son susceptibles de agruparse con el pronombre quien.

Cuyo: este es un pronombre que tiene características muy particulares que lo diferencian de los dos anteriores: en primer lugar, tiene variación de género y número y un significado de posesión. De ahí que la concordancia se establezca con ese objeto poseído y no con el objeto o la persona con la cual se relaciona como pronombre. Ejemplo: el estudiante cuyo promedio..... o el estudiante cuyas calificaciones... Observamos que el antecedente en ambas oraciones es estudiante y lo que ha variado es el objeto poseído; por tanto, el pronombre concierta con este elemento y no con su antecedente.

Cual: este pronombre nunca aparece solo como nexo de subordinada; siempre lo acompaña el artículo determinado y hace referencia a un antecedente femenino, masculino y hasta neutro. El pronombre como tal solo tiene variación de número, pero el artículo establece la concordancia según su antecedente sea masculino o femenino, singular o plural. Así, nos lo encontramos en contextos como el cual, la cual, los cuales, las cuales. Como en otros pronombres, hay una forma neutra marcada por el artículo neutro lo cual para referirse a ideas específicamente.

Por otra parte, el pronombre que puede también ir acompañado de los artículos definidos, y en este sentido adquiere un significado de generalización equivalente a quien, quienes: el que, la que, los que, las que y un neutro lo que para referirse a ideas complejas y que no puede ser sustituido por quien; pero a diferencia de cual y quien, el pronombre que puede ir acompañado de los demostrativos, en cuyo caso se habla de una sustantivación de la oración de relativo. Ejemplo: Aquel que está en la esquina.

Respecto del verbo de la subordinada de relativo, este puede estar en modo indicativo o en modo subjuntivo. Cuando el antecedente es conocido para el hablante, el verbo va en indicativo y cuando es desconocido, hipotético o no existe, va en subjuntivo. Ejemplo:

El estudiante que está es esa situación no puede hacer el examen.

El estudiante que esté en esa situación no puede hacer el examen.

En el primer caso, hay certeza, se conoce que el estudiante tiene una situación conflictiva que no le permite hacer el examen. En el segundo caso, no hay certeza, se enuncia como algo general que pueda que no sea cierto para todos.

Lo mismo sucede si se trata de acciones, ideas, estados o acontecimientos desconocidos o futuros en los que usamos la estructura neutra lo que para referirnos a ellos. En estos casos, el verbo también va en subjuntivo. Ejemplo: Haré lo que usted diga. A diferencia de Haré lo que usted dice.

Manuel Pérez Saldanya, en su exposición sobre "El modo en las subordinada relativas y adverbiales" (2000: 3255), afirma que "la relativa se construye con indicativo cuando el $\mathrm{SN}$ es específico y, por lo tanto, cuando la entidad que designa existe en un mundo concreto 
del universo del discurso; por el contrario, se construye con subjuntivo cuando el SN es inespecífico y la entidad no existe o el emisor no puede o no quiere afirmar su existencia".

Más adelante, el mismo autor hace un análisis semántico-sintáctico de estas oraciones en relación con el modo, en el que toma en cuenta aspectos más abstractos tales como el de la oposición asertivo/ no asertivo, para concluir que es necesario analizar el modo a partir del tipo de construcción sintáctica en el que se encuentra la oración de relativo. El modo subjuntivo es el de la no aserción y se caracteriza por el hecho de no afirmar la existencia del referente, bien porque no tiene una referencia específica o el hablante duda de ella o porque a pesar de ser específica no constituye el objetivo básico de la comunicación; mientras que el indicativo es el modo de la aserción y se caracteriza por afirmar la existencia del referente o por otorgar un carácter asertivo al contenido de la oración relativa en contextos en que el antecedente tiene una mención genérica y la relativa designa hechos habituales.

En relación con el aspecto sintáctico, el autor establece cuatro tipos de oraciones de relativo de los cuales solo analizaré dos, pues son los únicos que cumplen con la condición de nexos, establecida al inicio de la propuesta:

Oraciones que responden a la oposición específico vs. inespecífico. Estas oraciones son prototípicas y el modo subjuntivo se encuentra en correlación sintáctica con un elemento modal denominado modalizador o elemento creador de contextos opacos. El verbo típico es buscar. Ejemplo: Busco una casa que (tiene /tenga) un gran jardín.

Otros creadores de opacidad son los verbos modales, la estructura ser + atributo (ser necesario, ser posible), sustantivos que expresen necesidad, probabilidad, etc. La alternancia modal es posible cuando estos elementos asumen un valor deóntico (de obligación, necesidad o permiso), un valor epistémico (de probabilidad o eventualidad) o un valor dinámico (de capacidad: ser capaz o poder).

Ejemplos:

Puedes utilizar el carro que (está/esté) desocupado. (Deóntico de permiso).

Quizás sea el hombre que (viéramos/vimos) en el parque. (Epistémico).

Esa máquina puede lavar ropa que (es /sea) muy fina. (Valor dinámico).

Otro grupo de elementos modales que comparten el carácter dubitativo son las oraciones interrogativas, generales o parciales:

¿Has encontrado el carro que te (gusta/guste)?

¿Dónde encontraré el carro que (es /sea) más barato?

El futuro cuando tiene un valor epistémico de probabilidad y el condicional con un valor modal también se comportan como creadores de opacidad. Ejemplo:

Lo haré a la hora que (pueda/puedo) llegar

Si pudiera me compraría un carro que (tuviera /tiene) bolsas de aire

Finalmente, la negación y los inductores negativos constituyen el último grupo de elementos modales pero, a diferencia de los anteriores, no permiten la alternancia indicativo / subjuntivo. Al respecto, el autor aclara: "Cuando esta categoría incide sobre el SN, el antecedente 
nominal y la relativa denotan un conjunto vacío, no existencial y el único modo posible es el subjuntivo" (2002: 3262). Ejemplo: No aportó ninguna prueba que (tuviera /*tenía).

\subsection{Las oraciones de relativo y el subjuntivo de indeterminación}

Es otro contexto sintáctico en el que es posible la alternancia modal y se puede delimitar a partir de las siguientes propiedades:

- $\quad$ Cuando no existe ningún elemento modal explícito que establezca diferencias de ámbito.

- $\quad$ Cuando la oración de relativo no tiene antecedente y suele funcionar como sujeto.

- $\quad$ La alternancia modal afecta con frecuencia el valor de verdad de toda la oración y no únicamente la especificidad del $\mathrm{SN}$ en el que se incluye la relativa.

Ejemplos:

Quien no esté/está matriculado no puede asistir a clases.

La oración de relativo funciona como sujeto de la subordinante y, de acuerdo con Pérez Saldanya, no hay ningún elemento modal que pueda justificar el uso del subjuntivo y el subjuntivo afecta el valor de verdad de toda la oración al punto de que puede equipararse con una oración condicional. Al respecto, considero que, si bien no hay elemento sintáctico que justifique el uso del subjuntivo, sí está clara la razón semántica e incluso hipotética: se usa el subjuntivo cuando exista la posibilidad de que haya algún estudiante que no esté matriculado, porque todos deberían estarlo, mientras que se usa el indicativo no como una posibilidad sino como un hecho real: alguien no se matriculó y no puede estar en el curso.

Un último aspecto de la oración de relativo es su clasificación. Al inicio se dijo que esta oración funciona como un adjetivo. El adjetivo en su función calificativa puede especificar o explicar al sustantivo. Estas mismas funciones las desempeña la oración de relativo. La función de la oración especificativa o restrictiva es la de diferenciar al sustantivo del resto de sus iguales. Por ejemplo: El carro que compré el año pasado es bueno. La oración que compré especifica de cuál carro estoy hablando, es decir, lo diferencia del resto de carros. Siguiendo a Comrie, esta oración de relativo sirve para delimitar los referentes potenciales del antecedente; en este caso, el carro, debido a que el hablante supone que la oración el carro es bueno no proporciona al oyente la información necesaria para identificar el carro en cuestión; de manera que la información que compré el año pasado se añade para indicar de qué carro se está hablando. Por otra parte, cuando las oraciones de relativo cumplen la función de explicar el sustantivo, se trata de enfatizar una cualidad que ya tiene el sustantivo; por eso se llaman incidentales, pues se pueden suprimir sin afectar el significado de la oración. Por ejemplo: Estas flores, que son delicadas, se secan pronto. En este caso, la condición de delicadeza es inherente a esas flores en particular, es decir, expresa la causa, el incidente por el cual las flores se secan. En estas oraciones, según Comrie, el hablante supone que el oyente puede identificar de qué flores se está hablando y que son unas en particular e identificables. De acuerdo con Comrie (1981:200),

A pesar de que las construcciones de las oraciones de relativo restrictivas y no restrictivas son parecidas sintácticamente, son totalmente diferentes en términos pragmáticos o semánticos, sobre todo porque la oración de relativo restrictiva utiliza información presupuesta para identificar el referente de un sintagma 
nominal, mientras que la relativa no restrictiva es una forma de presentar nueva información basándose en que el referente ya puede identificarse. Sin embargo, desde el punto de vista tipológico esta distinción parece ser totalmente irrelevante.

\section{Estrategias metodológicas para enseñar las oraciones de relativo}

La parte metodológica tampoco está exenta de discusiones. Al igual que la polémica de si se enseña gramática o no en un curso de segundas lenguas, en este apartado se discute si la gramática debe enseñarse en forma explícita o implícita, es decir, con un método deductivo o inductivo. Hay innumerables propuestas y estrategias que apoyan a uno o a otro e, incluso, están las propuestas de combinar las dos metodologías. Particularmente, considero que hay temas en gramática que solo el especialista los puede explicar y probablemente en una clase magistral, lo cual no significa desconocer las metodologías participativas modernas. Hay temas que se prestan más para las metodologías participativas del tipo inductivas; sin embargo, lo más importante es enseñar y hacerlo bien.

En este apartado, se seguirán las propuestas del modelo de la Jerarquía Accesible de los Universales lingüísticos, citado por Cook, los principios básicos para la enseñanza de la gramática que propone Thornbury (1999:25) y el uso de la lengua total para la enseñanza de la gramática de Bonnie Adair-Hauck y otros (1994: 95).

La Jerarquía Accesible es un modelo para analizar desde el punto de vista de los universales lingüísticos las oraciones de relativo. Keenan y Comrie, citados por Cook (1996:27), descubrieron que había similitudes interesantes en la forma como las lenguas combinan la oración principal y la de relativo; estas características dependen de la función que la frase nominal cumple en la oración de relativo que fue usada como nexo con la oración principal. Tal concepto se llamó Jerarquía Accesible, puesto que algunas frases podían ser "accesadas" al hacer las oraciones de relativo unas más fáciles que las otras. Al respecto, Comrie explica: "En la discusión de este apartado subyace algo muy intuitivo y simple: la jerarquía de sujeto $>$ objeto directo $>$ objeto no directo $>$ poseedor define la escala de accesibilidad para formar oraciones de relativo, es decir, de forma intuitiva resulta más fácil relativizar el sujeto que ninguna otra posición; más fácil es relativizar el objeto directo que el poseedor, etc. Naturalmente, hay que verificar esta intuición, pero parece llevarnos a la formulación del siguiente universal lingüístico: si una lengua puede formar oraciones de relativo con una posición dada, en la jerarquía, también puede formar oraciones de relativo con todas las posiciones que estén más altas (a la izquierda) en la jerarquía, puede haber una lengua que pueda relativizar esa posición y todas las que están a la izquierda, pero no la posición que está a la derecha" (1981: 223). No obstante, el estudiante debe tener claro que en su lengua materna no necesariamente aparecen todos los tipos, lo que implicaría mayor dificultad de aprendizaje del tipo que está ausente en su lengua. La Jerarquía Accesible la constituyen seis tipos de oraciones de relativo y, según Cook, se pueden enseñar fácilmente como tipos del 1 al 6.

Primer tipo: oraciones ligadas al sujeto

Segundo tipo: oraciones ligadas al complemento directo

Tercer tipo: oraciones ligadas al complemento indirecto

Cuarto tipo: oraciones de caso oblicuo

Quinto tipo: oraciones ligadas al posesivo

Sexto tipo: oraciones ligadas al objeto de comparación 
De estos seis tipos de oraciones, en el texto (Poema) que se va a usar como modelo de enseñanza solo aparecen tres:

a) Una oración ligada al sujeto: Aquel que es firme en su corazón persevera en sus principios hasta la muerte.

b) Tres oraciones ligadas al complemento directo: Yo admiro al hombre que puede sonreír ante los problemas, que puede sacar fuerza de la desgracia y que en la reflexión crece en valentía.

c) Una oración ligada al posesivo: Aquel cuya conciencia aprueba su conducta, persevera en sus principios hasta la muerte.

Thornbury propone dos principios básicos para la enseñanza de la gramática que servirán como el criterio para la evaluación de los acercamientos prácticos que se presentan:

\section{El Factor $\mathbf{E}$}

Dentro de este concepto se engloban criterios como economía, naturalidad y eficacia.

Dado que el ocuparse de la gramática es solo una parte de las actividades que el profesor debe practicar, y que el tiempo de clase es corto, se hace necesario que cualquier enseñanza de la gramática que se realice sea lo más eficiente posible. De esta forma, el elemento economía constituye un factor importante. Se ha comprobado que la economía es un factor clave en la enseñanza de habilidades: enseñar lo que es prioritario parece ser más eficaz que cuando se pretende abarcar mucho. Hay que ser económico, dice Thornbury en la enseñanza de la lengua, en términos de planeamiento y recursos. Cuanto más se base el profesor en instrucciones, más confundido se siente el alumno. El factor naturalidad también juega un factor importante: cuanto más sencillos y naturales se presenten los ítemes, mayores ventajas tendrá el profesor y los estudiantes. Es una ventaja para el profesor, porque no tiene mucho tiempo para estar elaborando materiales complicados y al alumno se le facilita el aprendizaje. Finalmente el profesor debe preguntarse: ¿funciona todo lo anterior? Es ahí donde el factor eficacia entra en juego. ¿Cómo saber que el estudiante está aprendiendo? De hecho, se pueden hacer evaluaciones para medir el aprendizaje; no obstante, bastante se ha discutido y se discute sobre la eficacia de las evaluaciones por los factores que involucra. El autor propone que como un prerrequisito para el aprendizaje es la atención, la eficacia de una actividad gramatical puede medirse en parte con el grado de atención que se produce durante esa actividad. La eficacia dependerá en gran medida de la calidad y cantidad de la información contextual, de la explicación y de la revisión. Finalmente, entender sin memorización podría ser ineficaz; por tanto, la eficacia depende de cuán fácil de recordar sea una presentación. Pero para que sea fácil de recordar, se necesita de una eficaz motivación; de ahí la importancia de una buena escogencia de materiales y actividades de motivación y para trabajar en clase. De acuerdo con el autor, las tareas y los materiales, para que sean motivadores deben ser relevantes para sus necesidades, alcanzables y retadores.

\section{El factor A: Apropiado}

No todos los estudiantes son iguales. Una actividad que funciona para un grupo, puede que no funcione para otro, inclusive, puede que no funcione ni siquiera en el nivel individual. 
De esta manera, para que una actividad funcione, debe tomarse en cuenta lo apropiado de esa actividad. Para verificar la propiedad o lo apropiado de una actividad, se deben considerar los siguientes factores: la edad de los estudiantes, el nivel, el tamaño y la constitución del grupo, las necesidades que tienen, los intereses, los recursos y materiales disponibles, las experiencias de aprendizaje anteriores y las expectativas actuales, los factores culturales que puedan afectar su actitud, el contexto educacional: escuela privada o pública, en su país de origen o en el exterior.

Todos estos factores que el autor engloba bajo el concepto de apropiado son determinantes para el aprendizaje de una segunda lengua. Por ser muy obvios, no se van a definir aquí; sin embargo, servirán para evaluar la propuesta de enseñanza de la oración de relativo.

Bonnie Adair-Hauk y otros proponen un enfoque cíclico para la enseñanza de la lengua total denominado PACE, que consta de cuatro pasos: 1) Presentación: en este paso se presenta la lengua total, con algún material auténtico. En nuestro caso, usaremos un poema. 2) Atención: Aquí se enfoca la atención del estudiante en el aspecto o aspectos de la lengua que se quieren estudiar; en nuestro caso, las oraciones de relativo. 3) Co-construcción: los estudiantes y el profesor deben ser co-constructores de las explicaciones gramaticales. 4) Extensión: esta actividad provee a los estudiantes la oportunidad de poner en práctica su nueva habilidad de forma creativa e interesante, al mismo tiempo que la integra al conocimiento que existe. Los autores resumen los pasos en el siguiente esquema:

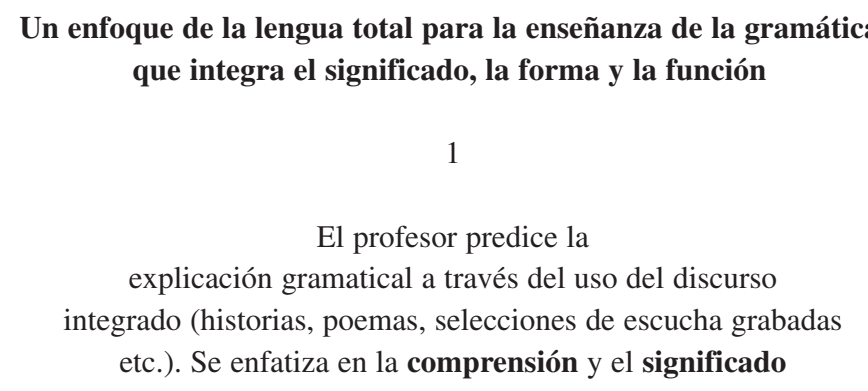

Por medio de actividades de extensión (actividades integradas que relaten la historia), los estudiantes necesitan usar las estructuras gramaticales para realizar una función o tarea

\author{
El profesor usa múltiples pasos y reconstruye \\ la historia a través de cuadros, TPR actividades \\ y juegos de representación que profundizan la \\ comprensión y aumentan la participación \\ del estudiante. De nuevo se enfatiza \\ el significado
}


Después de esta etapa, los autores aconsejan que el profesor complete el ciclo alentando a los estudiantes a interactuar con discursos integrados a través de la actividades de expansión tales como reescribir o recrear historias similares, actividades en pares o en grupo. Las sugerencias de cómo hacer estas actividades se describen seguidamente.

\section{Aplicación del modelo}

Tema: Enseñanza de las oraciones de relativo con verbo en indicativo

Nivel: Intermedio alto

\section{Actividades de presentación}

\section{Paso \#1}

El profesor les presenta a los estudiantes el siguiente poema de Thomas Paine. Lo lee en voz alta.

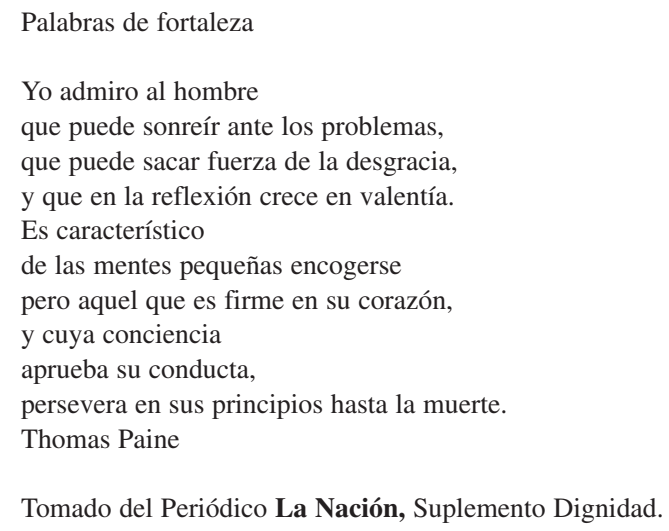

Paso \# 2

Le pide a algún estudiante que lo lea. Seguidamente les pregunta si hay algún problema de vocabulario para aclararlo.

\section{Paso \# 3}

El profesor les pide a los estudiantes que en grupos de tres discutan de qué se habla en el poema. 


\section{Actividades de atención}

Paso \# 1

El profesor anota en la pizarra las diferentes respuestas de los estudiantes; se discuten y entre todos escogen la que mejor representa el mensaje del poema.

\section{Co-construyendo una explicación}

Paso \#1

En las respuestas de los estudiantes se debe destacar la palabra hombre. Luego les pide a los estudiantes que le indiquen cómo se describe ese hombre en el texto, para que salgan las oraciones siguientes:

Hombre que puede sonreír

Hombre que puede sacar fuerza de la desgracia

Hombre que en la reflexión crece el valentía

\section{Paso \#2}

Seguidamente, les pregunta de qué otra forma se designa a ese hombre en el texto para que surja el pronombre aquel y les pregunta cómo se describe; ¿qué tiene en particular?

\section{Paso \# 3}

El profesor escribe las cinco oraciones subordinadas, numeradas y les pide a los estudiantes que indiquen qué tienen en común las cuatro primeras y qué sucede con la última.

Encierra en un círculo los pronombres relativos y pregunta si conocen esa palabra. Es muy probable que los estudiantes, por su nivel, ya conozcan los pronombre relativos, así la respuesta sería que se llaman pronombres relativos. Si no los conocen, el profesor tratará de que ellos, por lo menos, adivinen que son pronombres y luego enfatiza la presencia del verbo para llegar al concepto de oración de relativo; siempre con preguntas para que sean los estudiantes quieres participen identificando las oraciones y describiendo sus características.

\section{Actividades de extensión}

\section{Paso \#1}

Como actividad de extensión, una vez comprendido el uso y la estructura de estas oraciones, el profesor les pide a los estudiantes que mediante oraciones semejantes describan con tres características a alguna persona que ellos admiran. 


\section{Paso \#2}

El profesor le pide a cada estudiante que pase y escriba en la pizarra una de las oraciones que redactó. Se discute y se afianza el conocimiento de la nueva estructura.

\section{Paso \#3}

Como tarea, se les pide que escriban un poema en el que se destaquen otros valores del ser humano, tratando de usar los diferentes pronombre relativos. Se reparte otro texto con oraciones similares pero que algunas tengan el verbo en subjuntivo, para iniciar el mismo proceso en la próxima lección hasta completar el tema.

\section{Discusión}

El uso de un poema para enseñar un tema gramatical se considera material auténtico y tiene el privilegio de presentar la lengua en su uso real; no obstante, el profesor debe seleccionar muy bien el texto que va a usar. Este poema es fácil por cuanto es una exaltación de valores que son universales, que no están mediatizados por la cultura. En cada actividad se plantea una serie de pasos que el profesor debe seguir al pie de la letra. La primera regla es que los estudiantes entiendan el texto y para ello la aclaración del vocabulario resulta fundamental. Por tal motivo, las dos primeras actividades, a saber, Presentación y Atención, enfatizan el significado, se profundiza la comprensión y se promueve la participación de los estudiantes. En la actividad tercera, Co-construcción, se focaliza la forma y tanto el profesor como los estudiantes tratan de encontrar la explicación gramatical, para finalmente en la etapa cuarta aplicar el conocimiento aprendido elaborando textos en los que se usen las estructuras estudiadas. Como actividad de extensión, el profesor les da otro texto en el que además de las estructuras estudiadas el aprendiz se va a encontrar con otras estructuras en las que el verbo ha cambiado de modo y nuevamente se iniciará otro proceso de aprendizaje. Así, se cumple con la propuesta de Harmer (1997: cap.2) en la que el significado, la forma y la función deben interactuar en la enseñanza de la gramática.

\section{Evaluación}

De acuerdo con Thornbury, la propuesta cumple con los requisitos del factor E: eficiencia. Es una propuesta económica, natural y eficaz. Es económica pues se trata de un poema catalogado como un material auténtico de fácil lectura cuyo tema también es fácil de extraer. No se gasta mucho tiempo en su lectura ni es de gran complejidad para los estudiantes; por lo tanto, se cumple también con el factor eficacia, por la experiencia de una gramática que se aprende satisfactoriamente cuando tanto el profesor como los estudiantes co-construyen las explicaciones.

\section{El Factor A: apropiados}

Por ser un texto auténtico de poesía, se propone para estudiantes de nivel medio alto. En él se destacan valores universales del ser humano muy apropiados para estudiantes con 
cierta madurez. El texto no se circunscribe a una cultura particular y cumple con las expectativas del estudiante, pues como decía una vez un poeta costarricense: ¿Quién no ha escrito en su vida un poema? Por lo tanto, además de la variable edad, está acorde con el nivel y no tiene restricciones para el tamaño, las necesidades y los intereses del grupo. Cumple especialmente con la variable constitución del grupo, ya que, por tratarse de un universal lingüístico, todos los estudiantes la comprenderán con facilidad.

\section{Conclusiones}

En este trabajo se han combinado tres propuestas teórico-metodológicas para la enseñanza de las oraciones de relativo: por una parte, la Jerarquía Accesible, que es fundamentalmente para que el profesor tome en cuenta las variables del aprendiz en el sentido de cuál es su lengua materna y que, a su vez, el aprendiz pueda descubrir las semejanzas o diferencias en relación con esta; en este sentido, la Jerarquía Accesible no incide directamente en el desarrollo metodológico sino que sirve de fundamento teórico para la enseñanza de las oraciones de relativo. La propuesta de Bonie Adair-Hauck y otros respecto de la enseñanza de la lengua total permite una serie de ventajas al usar textos auténticos para que el estudiante, guiado por su profesor, pueda descubrir las estructuras que se desean aprender y co-construir junto con el profesor las explicaciones. Finalmente, los pasos y la evaluación de Scott Thornbury resultan de gran valor para verificar si los contenidos que se están enseñando y los objetivos de clase realmente se están logrando.

\section{Bibliografía}

Celce-Murcia Marianne. S.F. "Grammar Pedagogy in Second and Foreign Language Teaching”. Landmarks of American Language and Linguistics. V.2

Comrie, Bernard. 1981. Universales del lenguaje y tipología lingüística. Madrid: Editorial Gredos.

Cook, Vivian. 2000. Second Language Learning and Language Teaching. London: Hodder Headline Group.

Dair-Hauck y otros. 1994. "Using a Whole Language Approach to Teach Grammar. Chapter 6" Teacher's Handbook.

Harmer, Jeremy. 1997. Teaching and Learning Grammar. London: Longman Group UK Limited.

Pérez Saldanya, Manuel. 2000. "El modo en las subordinadas relativas y adverbiales". Gramática Descriptiva de la Lengua Española. Madrid: Espasa-Calpe S.A. 
Real Academia Española. 1986. Esbozo de una Nueva Gramática de la Lengua Española. Madrid: Espasa-Calpe.

Suplemento Dignidad. La Nación. 2000. San José, Costa Rica 24 de setiembre. Casa Editorial El Tiempo S.A.

Thornbury, Scott. 2002. How to Teach Grammar. Pearson Education Limited. England: Longman.

Westney Paul. 2000. "Rules and pedagogical grammar. Chapter 4". Perspectives on Pedagogical Grammar. New York: University of Cambridge. 